Volume 8, No.6, November -December 2019

International Journal of Science and Applied Information Technology

Available Online at http://www.warse.org/ijsait/static/pdf/file/ijsait11862019.pdf

https://doi.org/10.30534/ijsait/2019/118620198

\title{
A Review on Energy-Efficient Next Forwarding Node Selection for Non-Localization Routing Protocol in UWSNs
}

\author{
Ahmad M. Khasawneh ${ }^{1}$, Hayfa Y. Abuaddous ${ }^{1}$, Attaa Al-Khaleel ${ }^{2}$, Abdullah A.M AL Sokkar ${ }^{1}$, \\ Farah S. AISyouf ${ }^{1}$, Hidaya Abomteer ${ }^{1}$ \\ ${ }^{1}$ Faculty of Computer Science and Informatics, Amman Arab University, Amman, Jordan. \\ ${ }^{2}$ School of Electrical Engineering and Information Technology, German Jordanian University, Ma'daba, \\ Jordan.
}

\begin{abstract}
The Underwater Wireless Sensor Network (UWSN) is considered a promising technology for collecting valuable data from underwater areas, particularly for aiding military operations and environmental predictions. UWSNs consist of underwater sensor nodes that have limited energy and use acoustics for communication. Routing in underwater sensor nodes is one of the challenging issues in UWSNs because of the need to forward data packets with minimal energy consumption and a high packet delivery ratio. Selecting the next forwarding nodes is one of the key components of routing in UWSNs and has a direct effect on energy consumption and the packet delivery ratio. Therefore, this problem has gained much attention from the research community with the intent of enhancing the performance of UWSNs. This paper qualitatively reviews routing protocols for UWSNs, focusing on Energy-Efficient next-hop selection method and its strengths and weaknesses. A summary of the qualitative investigation is presented highlighting aims, the next-hop selection method, metrics, and priority considerations. A comprehensive investigation is carried out focusing on energy, link quality, void awareness, reliability, and shortest path characteristics. Finally, we discuss potential future research directions in UWSNs for forwarding node selection.
\end{abstract}

Key words: energy consumption; forwarding selection; routing; underwater wireless sensor network; void-aware

\section{INTRODUCTION}

Recently, underwater wireless sensor networks (UWSNs) have earned significant attention from researchers of wireless and network communities, due to the growing use of UWSN applications for a range of purposes including ocean monitoring, offshore exploration, controlling underwater mineral extraction, wildlife studies, and monitoring marine life [1]. Underwater wireless sensor nodes sense the data from down level of water and forward it to the sink node placed at the water's surface. The sink node forwards the data to the onshore data center for further processing. In routing protocols, each node selects either a candidate node or a set for candidate nodes to forward data to the next hop towards the sink node. The candidate nodes are selected as next-forwarding nodes based on different criterion and priorities. The criterion is the selected metrics used in the selection of the forwarding nodes. The priorities are determined based on the node's location or selected metrics. The highest priority node can forward data to a destination with minimum route cost [2]. The sender node selects the forwarder by calculating the route cost for its neighboring nodes. Then, the best-selected candidate forwards the data while the rest of the neighboring nodes discard the data.

Routing is one of the fundamental issues in UWSNs. Most of the studies on UWSNs concentrate on MAC and physical layers. Nevertheless, some studies have been conducted in the network layer; however, the study of this area is still in its initial stages [3]. The process of designing and implementing a routing protocol for UWSNs is challenging due to the harsh underwater environment, characterized by high error rate, long propagation delay, low bandwidth, and energy limitations [1,2]. Therefore, this study takes into account these challenges and limitations in order to analyze the efficiency of routing protocols in UWSNs.

The selection of the next forwarding nodes is one of the major decisions in the design of routing techniques for UWSNs and has a direct effect on the energy consumption and packet delivery ratio [4]. More precisely, the next 
forwarding nodes have been chosen based on the selected metrics. The best node forwards the data packets, and the rest of the nodes hold the data packets. The holding nodes forward the same data packets if the computed holding time finishes without overhearing the forwarding of the same data packets. Several forwarding nodes' selection algorithms have been suggested to reduce the energy consumption and increase the reliability in routing protocols for UWSNs [5]. Therefore, the design and development of reliable energy-efficient routing techniques are still being investigated to reduce the energy consumption and improve the packet delivery ratio.

In this context, this paper carries out a comprehensive review of routing algorithms for energy-efficient UWSNs, focusing on next-hop selection technology and challenging. The impact of the selection of next forwarding node on the assign of routing protocols in UWSNs is also highlighted we discuss each routing protocol, highlighting the energy-efficient next-hop node selection method and its strengths and weaknesses. We summarize and compare routing protocols for energy-efficient UWSNs based on the number of characteristics. The summary is based on different features including metrics, next-hop selection method, and priority for the best node. The comparison is based on the following performance factors: energy, link quality, void-aware, reliability, multi-hop, and shortest path. Lastly, we conclude this paper by discussing some important future issues that need to be taken into account when designing routing protocols for UWSNs.

\section{UNDERWATER WIRELESS SENSOR NETWORK}

In general, Underwater Wireless Sensor Networks (UWSNs) can be defined as a group of wireless sensor networks that are used to monitor underwater environments for various purposes including military, natural disaster prediction, and weather forecasting. Since UWSNs use acoustic links as a communication medium, they are also known as Underwater Acoustic Sensor Networks (UASNs). Some of the unique features of UWSNs, which are quite different from traditional Wireless Sensor Networks (WSNs), are discussed below. Firstly, most of the applications utilize three-dimensional network topology due to the three-dimensional nature of seas' and oceans' environment. More precisely, employing three-dimensional network topology in UWSNs requires a large number of sensor nodes. Therefore, underwater networks employ sparse network topology due to expensive underwater equipment, and the use of a vast number of nodes causing high network costs $[3,4]$. Secondly, the random movement in different depths and the free movement of sensor nodes by water currents in UWSNs causes dynamic network topology [1,5]. Moreover, the vertical movement has been reported to be negligible, while it is indicated that the horizontal movement is about $1-3 \mathrm{~m} / \mathrm{s}$ [2]; Thirdly, it is difficult to replace and charge the battery of sensor nodes given the harshness of underwater environments. This issue has a direct effect on energy efficiency in underwater environments [2,3]. Lastly, the use of a global positioning system (GPS) is not possible in underwater environments due to the $1.5 \mathrm{GHz}$ radio frequency used in GPS, which can be rapidly absorbed in water $[2,3]$.

\section{NEXT FORWARDING NODE SELECTION IN UWSNS}

In this section, next forwarding node selection for UWSNs is reviewed. Firstly, the impact of next forwarding node selection on the design of routing techniques is investigated. Secondly, the related literature is critically. Thirdly, a comparative investigation is performed, focusing on the strengths and weaknesses of the techniques considered.

\subsection{Impact of Next Forwarding Node Selection on the Design of Routing Techniques}

Next forwarding node selection is one of the most common issues in routing protocols that must be taken into consideration $[4,6]$. This issue attracts researchers to design a next forwarding node algorithm. In routing, the general structure of this algorithm is as follows. Each source node selects a group of its neighbors. These nodes have been chosen based on different metrics such as residual energy, physical distance, and link quality. The nodes then hold the data packet for a certain length of time based on various criteria such as distance, sound propagation speed, transmission range, and residual energy to avoid collision and redundant packet transmission. The best node has the lowest holding time, so it has the highest chance of forwarding the data packets. If a node overhears the forwarded data packet, it simply removes the data packet from its buffer. Otherwise, it waits until its holding time finishes to forward the packets. Therefore, these algorithms have a direct impact on performance indicators such as packet delivery ratio and total energy consumption.

The use of different metrics for selecting the next forwarding node has a direct impact on the overall performance of routing protocols. The use of a residual energy metric helps in balancing the energy between nodes. Link quality is another important metric that has a direct impact on improving the packet delivery ratio and reducing 
energy consumption [6]. The use of a depth metric helps in reducing the energy consumption because each node calculates the depth locally, whereas the physical distance can be calculated using beaconing messages provided by the sink. The use of GPS for identifying the location of the node is costly due to the inability to use GPS in underwater environments. Therefore, it is essential to design a forwarding node selection algorithm based on multi-metrics, which selects energy-efficient and reliable forwarding nodes to reduce energy consumption, reduce network traffic, and ensure data delivery.

In this paper, we focus on location information and forwarding techniques as selected criteria to introduce review of energy-efficient forwarding node algorithms in UWSNs. Routing protocols have been divided into two categories: localization and non-localization routing protocols. Each category has been divided into different subcategories based on the selection mechanism of forwarding nodes. Some of the protocols belong to a different category. The next sections describe the features of energy-efficient non-localization category and some existing well-known routing protocols in detail, along with techniques used in these protocols to handle forwarding node selection.

\subsection{Non-Localization Routing Protocols}

Non-localization routing protocols did not fully use location information for selecting the next forwarding nodes during the routing process. To find the group of forwarder nodes toward the sink, routing protocols in this category use other information such as physical distance, hop-count, layering, dynamic address, and depth of nodes. Non-localization category is divided according to data collection methods into two subcategories: beacon-based and pressure-based. In beacon-based, a group of next forwarding nodes toward the sink is identified based on special information about the network such as addressing based and physical distance that is provided by sending periodic beacon messages from sink nodes to the ordinary nodes from the surface of the water to the bottom.

On the other hand, depth information is the main factor used in the pressure-based subcategory to find the position of each node. All of the nodes are equipped with inexpensive pressure sensors that can calculate the depth manually and locally by changing the pressure of the water in different depths in the water current. The next forwarding node is the node that has less depth than the sender node. This process is continuously repeated in a hop-by-hop manner until reaching the sink node located on the water surface in UWSNs. The pressure-based method does not need expensive and full location information compared to the location-based and beacon-based methods. The location-based category needs complete information about a node's location, and the beacon-based category requires sending expensive beacon messages. Conversely, a pressure-based category just employs depth information that is obtained locally without high cost and extra overhead.

Energy Efficiency: As mentioned before, energy efficiency is one of the main goals that attracts researchers designing routing protocols. In this subcategory, the main aim is to reduce the energy consumption and improve the network lifetime by using residual energy factor or some of the factors that help balance the energy consumption between sensors. The following paragraphs discuss the localization routing protocols belonging to this subcategory in detail.

The Depth-Based Routing (DBR) Protocol is considered as a first pressure routing that provides scalable and efficient routing services in UWSNs [7]. This protocol does not need full location information for the sensor nodes; it only requires local depth data. In this protocol, in order to select the next forwarding nodes, the sender node tries to find the best neighboring nodes regarding depth information to forward the packet. In other words, neighboring nodes with less depth than the sender will be candidates for forwarding the packet, as shown in Figure 1. DBR follows a specific routing procedure, starting with the sender node broadcasting the packets, including its depth information, to its one-hop neighbors. After receiving the packets, the neighboring node compares its depth with the sender's depth, which is embedded in the packet. This node will be a candidate for forwarding the packet if its local depth is less than the sender depth. Otherwise, the node directly discards the packet. After that, the candidate nodes broadcast the packet, including their depth, to their one-hop neighbor nodes, and so on. This technique uses hop-by-hop. In each hop, the data packet is sent to a node with a lower depth than the sender until it is delivered the sink. Moreover, for each received packet, each forwarder node calculates the holding time based on the depth information. The candidate nodes start forwarding the packets if the holding time finishes. Therefore, the node with the lowest depth is the best sender node. Upon overhearing the packet, the candidate node removes the packet from its buffer. Otherwise, it will forward the packet after its holding time is finished.

DBR has some advantages. The employment of multi-sink helps reduce the energy consumption for nodes placed closer to the sink. Moreover, it does not require any location information as it calculates the depth locally. However, DBR does not provide efficient next forwarding 
node selection due to the lack of energy and reliable metrics. It uses only depth information in terms of forwarding and holding the data packet. Moreover, it still does not provide any solution to the communication void problem. Furthermore, it selects the shortest path based on depth information only, which has a direct impact on reducing the energy consumption due to always selecting the node with the lowest depth.

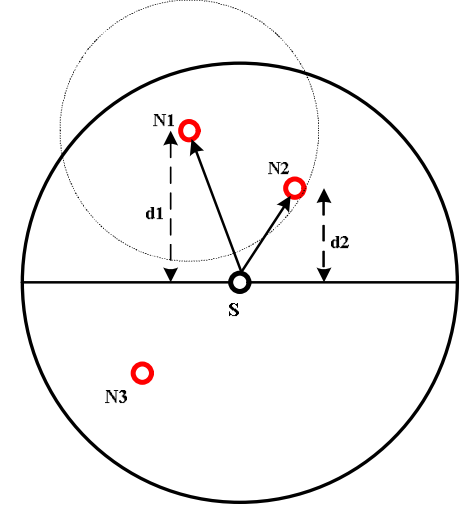

Figure 1: Packet forwarding process in DBR

Based on DBR, a new routing protocol called Depth-Based Multi-Hop Routing Protocol (DBMR) has been proposed [8]. This protocol tackles an energy-efficient routing problem in an underwater sensor network by using the multi-hop mode of each node to send packets, thereby reducing the communication cost. In addition, this protocol takes advantage of multiple sinks without introducing extra cost. DBMR consists of two phases, namely the route discovery phase and sending packets. In the route discovery phase, the process of selecting the next forwarding nodes has been designed as follows: each node discovers the next hop node by calculating its depth. Then, it broadcasts its ID and depth information as a control message. Next, it waits for a specific length of time to receive the reply message. After receiving the control message, the neighboring node will compare its depth with the depth included in the control message. The neighboring node will reply and forward the packet if its depth is less than the depth listed in the control message, as shown in Figure 11. This reply message consists of its weight (depth information and residual energy of the node) and ID using the equation $F(I D)=E r / d r$, where $d r$ is the depth of the current node and Er signifies the residual energy; otherwise, it will discard the message. After that, the largest weight $(F(I D))$ node will be selected as the next hop by each node when the holding time is over and stored in the routing table. In the sending packets phase, sending the packet to the sink requires gathering information about the next hop node from the routing table and choosing the best one. Then, the data packet is transmitted to the selected node directly to avoid communication overhead.

The advantages of DBMR are highlighted as follows. The employment of multi-sink helps reduce the energy consumption for nodes placed closer to the sink. Moreover, the network lifetime has been improved because of the use of single-hop, next forwarding nodes selection. Furthermore, the use of residual energy during weight calculation has a direct impact on balancing the energy consumption. However, the key drawbacks of DBMR are: first, due to the lack of an efficient retransmission mechanism in DBMR, selecting only one next hop entails high energy consumption and end-to-end delay. Second, calculating the weight in DBMR is not efficient because it uses only residual energy metrics without taking into account link quality metrics, which promote packet loss. Third, DBMR suffers from the lack of a communication void avoidance algorithm. Last, the discovery phase should be updated with short intervals, leading to an increase in the network overhead.

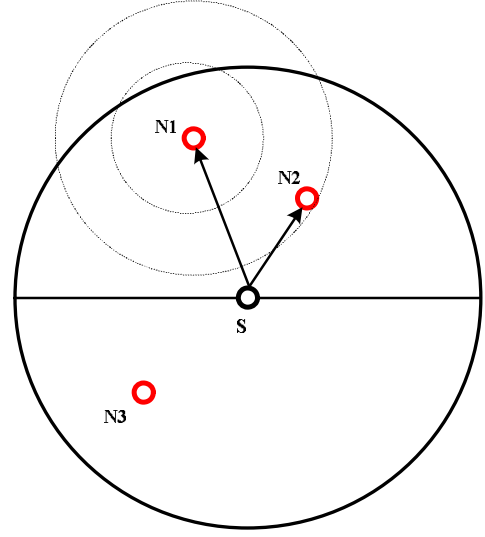

Figure 2: Route discovery phase in DBMR.

Energy-Efficient Localization-Free Routing (EEDBR) Protocol is an enhancement of DBR aimed at improving the energy efficiency in UWSN [9]. Because of the replacement of the batteries, energy is one of the key issues in designing a routing protocol. For this purpose, an energy-efficient routing protocol has been proposed, called Energy-Efficient Localization-Free Routing Protocol (EEDBR). In terms of forwarding data packets, EEDBR utilizes the depth of the sensor nodes. Moreover, it takes into account the residual energy of the sensor nodes to improve network lifetime and reduce the energy consumption. EEDBR is divided into two phases, namely knowledge acquisition and data forwarding. During the knowledge acquisition phase, the next forwarding node is selected. To this end, each node broadcasts a hello packet including its local depth information and residual energy to its neighbor nodes. Only the nodes that have less depth than 
the sender nodes store the data in their tables, sorted based on residual energy then depth information. In the data forwarding phase, the sender node broadcasts the packet, including the nodes' ID, to its neighbors. Each node that receives the packets extracts the ID. If the node matches its ID with the ID embedded in the packets, it will calculate the holding time based on residual energy using the following equation:

$$
T=\left(1-\left(\frac{\text { curront onsegy }}{\text { hitial energy }}\right)\right) \times \text { max_holdtng_time }+p \text {, }
$$

Where max_holding_time refers to the maximum holding time and $p$ is the priority value. Then, it forwards the data packets after a certain holding time. Otherwise, it discards the packet. If the node overhears the packets, it generates a random number and compares it with the delivery ratio; if this number is less than the delivery ratio, it will drop the packets; otherwise, it will forward the data packets after the holding time finishes. On the other hand, if the node does not overhear the packets, it will forward the data packets after its holding time finishes.

The main advantage of EEDBR is that it employs multi-sink, which has a direct impact on balancing the energy between the nodes that are closer to the sink. Moreover, it uses local depth information only, without any expensive GPS or beacons. However, the disadvantages of EEDBR are discussed as follows. EEDBR utilizes only residual energy for selecting the next forwarding nodes and does not use any link quality metrics, which does not ensure energy-efficient and reliable routing. Moreover, it selects the node that has less depth than the sender based on residual energy, ignoring the depth of neighbors, which leads to an increase in the number of retransmissions and the number of hops. Furthermore, the knowledge acquisition phase should be updated with a short interval of time, which leads to high network overhead. Lastly, EEDBR does not take into account the communication void, which is considered a critical issue in selecting the next forwarding nodes.

Based on layering, Localization-Free Multi-Layered Routing Protocol (MRP) has been proposed by [10]. The author has introduced a new architecture based on Super Nodes. Super Nodes are connected directly with sink nodes placed on the water surface, as illustrated in Figure 3. Ordinary nodes are deployed at the bottom level of the water. These super nodes divide the area into layers using beacon messaging based on different transmission power. Thus, selecting the next forwarding nodes is based on layer ID (layer-by-layer), and each node calculates the holding time based on residual energy until reach super nodes. All the nodes that overhear the same packet will drop this packet and so on.

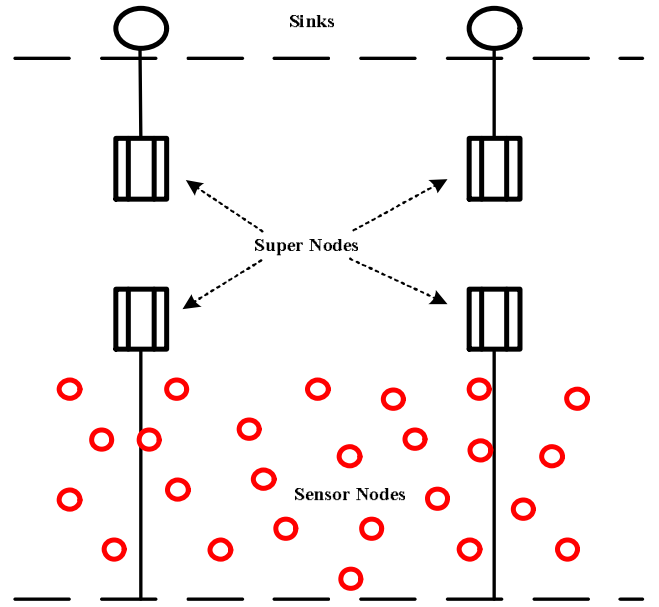

Figure 3: The use of super nodes in MRP.

MRP is related to $\mathrm{H} 2-\mathrm{DAB}$ in that it uses the same layering technique. Moreover, layer ID in MRP is equivalent to Hop-ID in H2-DAB. It produced a better result than H2-DAB. Moreover, MRP solves communication void problem by introducing a new architecture which has been designed based on super nodes. However, MRP suffers from some problems. The proposed architecture consumes high energy consumption due to the use of super. Moreover, the super node uses max power to send the data packets to another Super Node that placed above. Thus, it results in high energy consumption. Furthermore, MRP did not take the link quality parameter into its account which has a direct effect on packet delivery ratio.

\subsection{Comparative Analysis and Discussion}

Due to the unique characteristics and harshness of an underwater environment, Opportunistic Routing is one of the most interesting routing techniques in UWSNs. Based on forwarding nodes selection, routing protocols can be divided into two categories, localization and non-localization routing protocols. However, the routing protocols in the localization category are not efficient. This is because finding location information in this category is costly due to the lack of GPS in an underwater environment. Therefore, the non-localization category is the most interesting for designing routing protocols for UWSNs. Based on data collection, non-localization routing protocols can be divided into two major subcategories, beacon-based and pressure-based. On the other hand, in a pressure-based subcategory, the nodes are equipped with pressure sensors that are inexpensive and can calculate the depth information locally without using full or partial location information. This information can be employed for identifying the node's position and the next forwarding nodes without imposing any extra network overhead. More precisely, finding the next forwarding 
nodes in the pressure-based subcategory does not require full location information or partial sink information. Therefore, the opportunistic pressure-based routing subcategory is the most promising and interesting routing method for UWSNs. Hence, this study focuses on designing an energy-efficient and reliable opportunistic pressure-based routing protocol for UWSNs.

In this paper, we have reviewed the most common routing protocols in energy-efficient UWSNs regarding selecting the forwarding nodes. We have summarized the existing well-known routing protocols in Table 1 . In this table, the comparison between various routing protocols in UWSNs is simplified. The significance of each column is explained in the following.

- Category and Protocol: this field identifies our introduced categories and the names assigned by authors to the proposed protocols belonging to each category. The corresponding references are also provided here.

- Objectives: this field shows the main goal of the proposed algorithms, such as energy efficiency, reliability, and void handling.

- Modeling parameters: we have extracted the main metrics utilized in the existing routing protocols; each protocol uses different metrics in terms of selecting the next forwarding nodes.

- Neighbor selection strategy: the aim of this paper is to analyze the main issues in UWSNs regarding selecting the next forwarding nodes. Therefore, this column highlights the main techniques of how each protocol selects the next forwarding nodes.

- Forwarder selection strategy: this column points out how each protocol selects the best nodes among the candidates. Table 2 provides full performance factors of the protocols, which includes a summary of the main behaviors of all protocols discussed above. This summarization is based on dependent factors used in selecting the next forwarding nodes, including residual energy, link quality, void-aware, Table 2: Comparison of the main factors in non-localization routing protocols in energy efficiency UWSNs.

\begin{tabular}{|c|c|c|c|c|c|c|c|}
\hline $\begin{array}{l}\text { Pro } \\
\text { toc } \\
\text { ol }\end{array}$ & $\begin{array}{l}\text { Resi } \\
\text { dual } \\
\text { Ene } \\
\text { rgy }\end{array}$ & $\begin{array}{l}\mathbf{L i} \\
\mathbf{n k} \\
\mathbf{Q} \\
\text { ual } \\
\text { ity }\end{array}$ & $\begin{array}{l}\text { Vo } \\
\text { id- } \\
\text { A } \\
\text { wa } \\
\text { re }\end{array}$ & $\begin{array}{l}\text { Reli } \\
\text { abili } \\
\text { ty }\end{array}$ & $\begin{array}{l}\text { Mul } \\
\text { ti-h } \\
\text { op }\end{array}$ & $\begin{array}{l}\text { Sh } \\
\text { ort } \\
\text { est } \\
\text { Pa } \\
\text { th }\end{array}$ & $\begin{array}{l}\text { Sender/ } \\
\text { Receive } \\
\text { r-Base } \\
\text { d }\end{array}$ \\
\hline $\begin{array}{l}\mathrm{DB} \\
\mathrm{R} \\
\mathrm{DB} \\
\mathrm{MR}\end{array}$ & $\checkmark$ & & & & $\checkmark$ & $\checkmark$ & $\begin{array}{l}\text { Receive } \\
\text { r-based } \\
\text { Sender- } \\
\text { based }\end{array}$ \\
\hline
\end{tabular}

reliability, multi-hop, shortest path, and sender/receiver-based.

Table 1: Features of non-localization routing protocols in UWSNs in energy efficiency of selecting the next forwarding nodes.

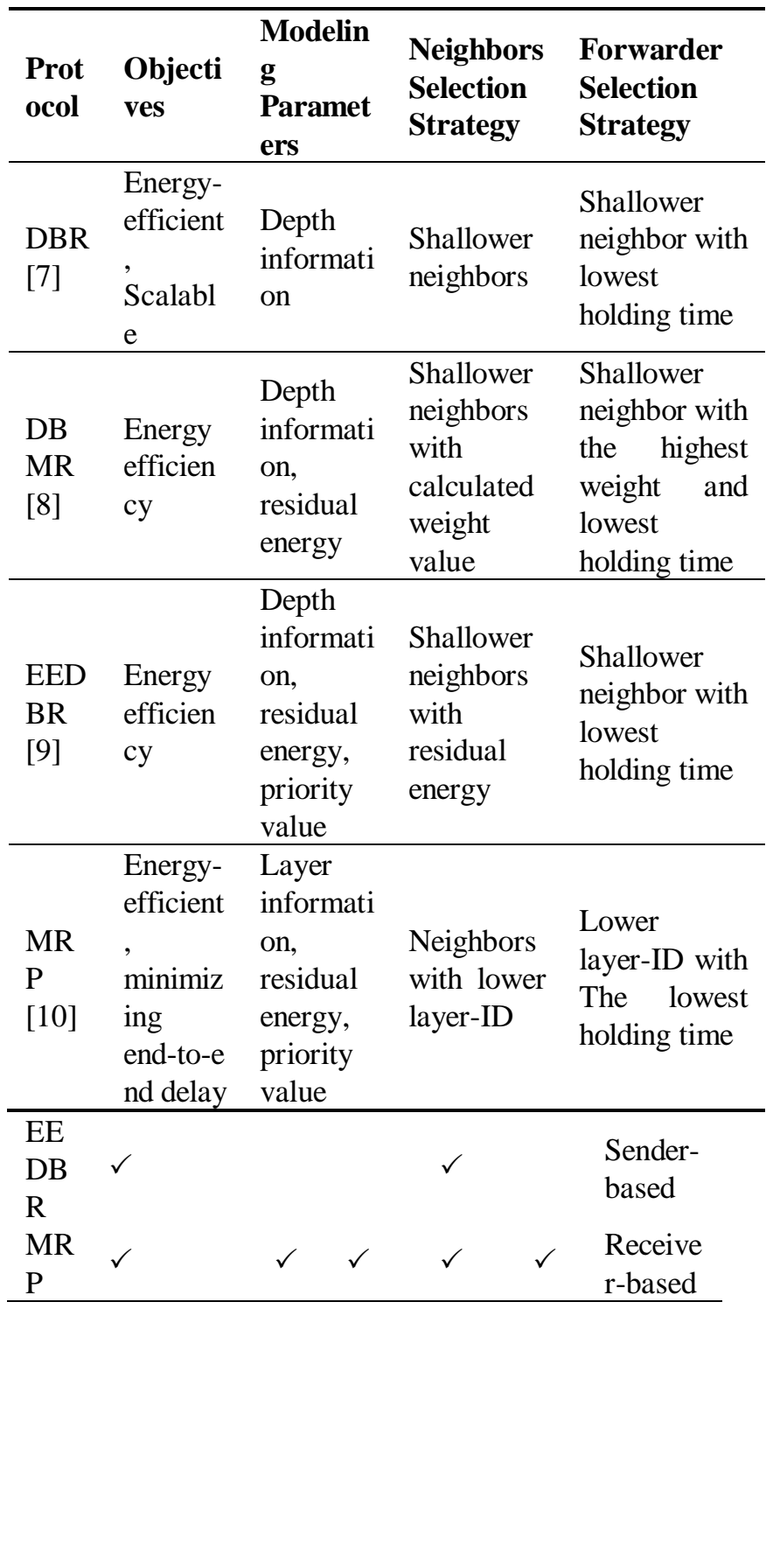




\section{FUTURE ISSUES IN ENERGY-EFFICIENT NEXT FORWARDING NODE SELECTION IN UWSNS}

Forwarding nodes selection in UWSNs is a major challenge due to the use of acoustic waves, a communication medium that causes high energy consumption and low packet delivery ratio. Based on our comparative analysis and discussions, the future issues in next forwarding nodes selection can be listed as follows.

- Energy-Efficiency: The use of different metrics during the process of selecting the next forwarding nodes has a direct impact on the overall performance of the protocol $[1,6]$. Therefore, it is essential to design and develop an energy-efficient and reliable forwarding nodes selection algorithm based on residual energy and suitable link quality, to balance the energy consumption, improve the delivery ratio, and further optimize the network lifetime [6].

- Route cost calculation: as discussed previously, an efficient cost calculation has a direct impact on selecting the best node among neighbors [1,2]. Therefore, convenient route cost should be carefully designed using suitable metrics such as depth, link quality, and residual energy, which in turn improve the network lifetime and reduce network overhead.

\section{CONCLUSION}

Energy-Efficient next forwarding nodes selection is one of the fundamental issues in the design of routing protocols. This issue spurs researchers to design efficient and effective next forwarding node selection methods. In this paper, have comprehensively reviewed important and representative protocols in the selected literature. We have discussed each routing protocol, highlighting the next-hop selection method with its advantages and disadvantages. We have compared and summarized the routing protocols, considering their features and performance metrics. Finally, we have pointed out some important future research directions that need to be investigated further to develop an efficient next-hop selection method for routing in UWSNs.

\section{REFERENCES}

1. Khasawneh, A., Latiff, M.S.B.A., Chizari, H., Tariq, M. and Bamatraf, A., 2015. PRESSURE BASED ROUTING PROTOCOL FOR UNDERWATER WIRELESS SENSOR NETWORKS: A SURVEY. KSII Transactions on Internet \& Information Systems, 9(2). https://doi.org/10.3837/tiis.2015.02.002

2. Khasawneh, A., Latiff, M.S.B.A., Kaiwartya, O. and Chizari, H., 2017. Next forwarding node selection in underwater wireless sensor networks (UWSNs): Techniques and challenges. Information, 8(1), p.3.

3. Wahid, A., Lee, S. and Kim, D., 2014. A reliable and energy $\square$ efficient routing protocol for underwater wireless sensor networks. International Journal of Communication Systems, 27(10), pp.2048-2062.

4. Ghoreyshi, S., Shahrabi, A. and Boutaleb, T., 2016. A novel cooperative opportunistic routing scheme for underwater sensor networks. Sensors, 16(3), p.297.

5. Coutinho, R.W., Boukerche, A., Vieira, L.F. and Loureiro, A.A., 2016. Design guidelines for opportunistic routing in underwater networks. IEEE Communications Magazine, 54(2), pp.40-48.

6. Khasawneh, A., Latiff, M.S.B.A., Kaiwartya, O. and Chizari, H., 2018. A reliable energy-efficient pressure-based routing protocol for underwater wireless sensor network. Wireless Networks, 24(6), pp.2061-2075.

7. Yan, H., Shi, Z.J. and Cui, J.H., 2008, May. DBR: depth-based routing for underwater sensor networks. In International conference on research in networking (pp. 72-86). Springer, Berlin, Heidelberg.

8. Guangzhong, L. and Zhibin, L., 2010, May. Depth-based multi-hop routing protocol for underwater sensor network. In 2010 The 2nd International Conference on Industrial Mechatronics and Automation (Vol. 2, pp. 268-270). IEEE.

9. Wahid, A. and Kim, D., 2012. An energy efficient localization-free routing protocol for underwater wireless sensor networks. International journal of distributed sensor networks, 8(4), p.307246.

10. Wahid, A., Lee, S., Kim, D. and Lim, K.S., 2014. MRP: A localization-free multi-layered routing protocol for underwater wireless sensor networks. Wireless personal communications, 77(4), pp.2997-3012.

11. Abualigah, L. M. Q. (2019). Feature selection and enhanced krill herd algorithm for text document clustering. Berlin: Springer. 